\title{
Tailor-made Functional Polymers for Energy Storage and Environmental Applications
}

\author{
Ali Coskun*
}

\begin{abstract}
CO}_{2}$ emissions into the atmosphere account for the majority of environmental challenges and its global impact in the form of climate change is well-documented. Accordingly, the development of new materials approaches to capture and convert $\mathrm{CO}_{2}$ into value-added products is essential. Whereas the increased availability of renewable energy is curbing our reliance on fossil fuels and decreasing $\mathrm{CO}_{2}$ emissions, the widespread adaptation of renewable energy still requires the development of high energy density batteries i.e., lithium ion batteries (LIBs). To address these energy and environmental challenges, our group has been developing porous organic polymers (POPs) with precise control over their porosity and surface chemistry for $\mathrm{CO}_{2}$ capture, separation and conversion. To realize simultaneous $\mathrm{CO}_{2}$ separation and conversion, we are also developing catalytically active two-dimensional membranes and POPs. In the area of LIBs, we have recognized the potential of supramolecular chemistry as a general strategy for solving the capacity-fading problem associated with high energy density electrode materials such as Li-metal, silicon and sulfur, which offer extremely high battery capacity compared to conventional LIBs. Accordingly, we have demonstrated how molecular-level design of one- and two-dimensional supramolecular polymers can be directly translated into an improved electrochemical performance in high energy density LIBs.
\end{abstract}

Keywords: Carbon capture $\cdot$ Energy storage $\cdot$ Li-ion batteries $\cdot$ Porous materials $\cdot$ Supramolecular polymers

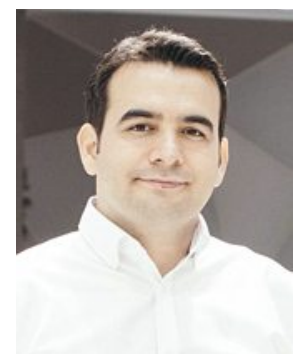

Ali Coskun was born in Isparta, Turkey. He received his MSc and $\mathrm{PhD}$ degrees in chemistry at the Middle East Technical University (METU) while carrying out his research activities under the supervision of Professor Engin U. Akkaya. During his graduate studies, he developed supramolecular ion-sensing systems and investigated their application in the area of molecular logic gates. After his graduate studies, he joined the laboratory of Prof. J. Fraser Stoddart as a postdoctoral research associate at Northwestern University, where he developed dynamic metal-organic frameworks, artificial molecular machines, organic radicals and molecular electronic devices based on switchable mechanically interlocked molecules. In 2012, he started his independent career as an assistant professor at the Graduate School of EEWS and Department of Chemistry in the Korea Advanced Institute of Science and Technology (KAIST) and was promoted to the rank of Associate Professor in 2015. In 2017, he moved to University Fribourg, Switzerland as a Professor, where his research is supported by SNSF. His group develops porous organic polymers for $\mathrm{CO}_{2}$ capture, separation and conversion, two-dimensional membranes, porous carbon materials for gas and energy storage, one- and two-dimensional supramolecular polymers for high energy density electrodes in Li-ion batteries.

\section{Introduction}

The consumption of fossil fuels to meet our ever-increasing energy demand is being challenged by growing concerns over anthropogenic emissions of greenhouse gases such as $\mathrm{CO}_{2}$, which

${ }^{*}$ Correspondence: Prof. A. Coskun, E-mail: ali.coskun@unifr.ch

Laboratory of Functional Organic Materials (LFOM), Department of Chemistry,

University of Fribourg, Chemin du Musée 9, CH-1700 Fribourg, Switzerland is accelerating global climate change and ocean acidification. ${ }^{[1]}$ Current atmospheric levels of $\mathrm{CO}_{2}$ average around $408 \mathrm{ppm}$, depending on the measuring location. ${ }^{[2]}$ The trend, however, is a steady increase over the past century. The global carbon cycle spins off an excess of 3.3 gigatons of $\mathrm{CO}_{2}$ per year that is unaccounted for ${ }^{[3]}$ This overflow is widely believed to be the major factor for the elevation in the atmospheric concentration of $\mathrm{CO}_{2}$. The sources of these emissions are known to be predominantly from fossil fuel combustion. Once released into the atmosphere, it becomes much more expensive and difficult to capture $\mathrm{CO}_{2}$ since the concentration in flue gases is about 300 times higher than it is in the air. ${ }^{[4]}$ The regulation of the carbon dioxide emissions suggests the necessity to develop specific $\mathrm{CO}_{2}$ capture technologies that can be retrofitted to existing power plants and designed into new plants with the goal to achieve $90 \%$ of $\mathrm{CO}_{2}$ capture while limiting the increase in the cost of electricity to no more than $35 \%$. The cost analysis of carbon capture and separation (CCS) revealed that the $\mathrm{CO}_{2}$ capture process alone accounts for $80-90 \%$ of the cost with the rest accounted for by transport and storage. ${ }^{[5]}$ Therefore, the low-cost and efficient recovery of $\mathrm{CO}_{2}$ from large emission sources is a formidable technological and scientific challenge, which has received considerable attention for several years. ${ }^{[6]}$ Presently, the most broadly implemented method is absorption using aqueous amine solutions (e.g. monoethanol amine), and this technology has been used in the natural gas industry for more than 60 years. The aqueous basic solvents selectively absorb the acidic $\mathrm{CO}_{2}$ at ambient conditions. Once saturated, the mixture is heated up for regeneration at temperatures well above $100^{\circ} \mathrm{C}$, which itself is a quite energy intensive process and presents additional problems such as amine degradation and corrosive nature of amine solutions. ${ }^{[7]}$ In this context, porous materials such as metal-organic frameworks (MOFs), zeolites, mesoporous silica, covalent organic frameworks (COFs) and porous organic polymers (POPs) have been extensively studied as possible alternatives for $\mathrm{CO}_{2}$ capture. ${ }^{[4]}$ The separation and capture of $\mathrm{CO}_{2}$ is only the first step in the fight 
against greenhouse gas emissions. The next and the most daunting challenge is the fate of the collected gases. ${ }^{[8]}$ Safe storage in a planetary void is still heavily debated and the $\mathrm{CO}_{2}$ market (if recycling is intended) is highly limited when compared with the massive scale of the emissions (less than 1\%). ${ }^{[9]}$ Evidently, there is almost no choice but to come up with safer, economically viable products (Fig. 1) from $\mathrm{CO}_{2}$ via effective conversions.[10]

Ideally, the systems capable of simultaneous separation and conversion of $\mathrm{CO}_{2}$ i.e., catalytic membranes, could reduce the overall costs of the process. Nevertheless, considering the massive scale of $\mathrm{CO}_{2}$ emissions, the widespread utilization of renewable energy is crucial to reduce this gap. The intermittent nature of renewable energy sources, however, require high energy density energy storage systems such as Li-ion batteries. In this direction, Li metal and silicon as anode and sulfur as cathode materials have emerged as promising candidates as they offer significantly higher capacities compared to the conventional graphite-based LIBs.[11] In order to tackle these energy and environmental challenges, Laboratory of Functional Organic Materials (LFOM) focuses on (1) the development of POPs and two-dimensional (2D) membranes with precise control over porosity, surface chemistry and catalytic activity to achieve $\mathrm{CO}_{2}$ capture, separation and conversion and (2) the development of supramolecular polymers to address the capacity fading problems associated with high energy density electrode materials in Li-ion batteries (LIBs).

\section{Porous Materials for $\mathrm{CO}_{2}$ Capture, Separation and Conversion}

POPs have emerged as promising candidates for $\mathrm{CO}_{2}$ capture, separation and conversion applications due to their high surface areas, low-cost, physicochemical stability, and tunable surface chemistry. Notably, synthesis of POPs is highly modular and the nature of monomeric units can dictate the function of the resulting POP. The presence of micropores $(<2 \mathrm{~nm})$ along with heteroatoms enables selective targeting of $\mathrm{CO}_{2}$ via dipole-quadrupole interactions. Likewise, POPs can also be engineered to have catalytic sites for $\mathrm{CO}_{2}$ conversion. In this direction, our group has developed series of POPs, MOFs and two-dimensional membranes for $\mathrm{CO}_{2}$ capture, separation and conversion applications with a primary focus on sustainable synthetic and materials approaches.

\subsection{POPs for $\mathrm{CO}_{2}$ Capture and Separation}

Our research in this area focuses on the development of sustainable bottom-up synthetic strategies without using any precious metal catalysts to achieve precise control over porosity and surface chemistry of POPs to tune their affinity towards $\mathrm{CO}_{2}$. In this direction, we developed (Fig. 2) a new class of porous materials called azo-bridged porous organic polymers (azo-COPs) for efficient $\mathrm{CO}_{2}$ capture and separation.

Unlike any previously reported porous material, azo-COPs showed an increase in $\mathrm{CO}_{2} / \mathrm{N}_{2}$ selectivity with rising tempera-
Fig. 1. The use of $\mathrm{CO}_{2}$ as a chemical feedstock and comparison of the $\mathrm{CO}_{2}$ market with total anthropogenic emissions.
Fig. 2. Synthetic strategy for the preparation of azo-linked porous organic polymers and their surface areas and corresponding gas $\mathrm{CO}_{2} /$ $\mathrm{N}_{2}$ selectivities at different temperatures. Figure reproduced with permission of the Publisher. ${ }^{[12]}$

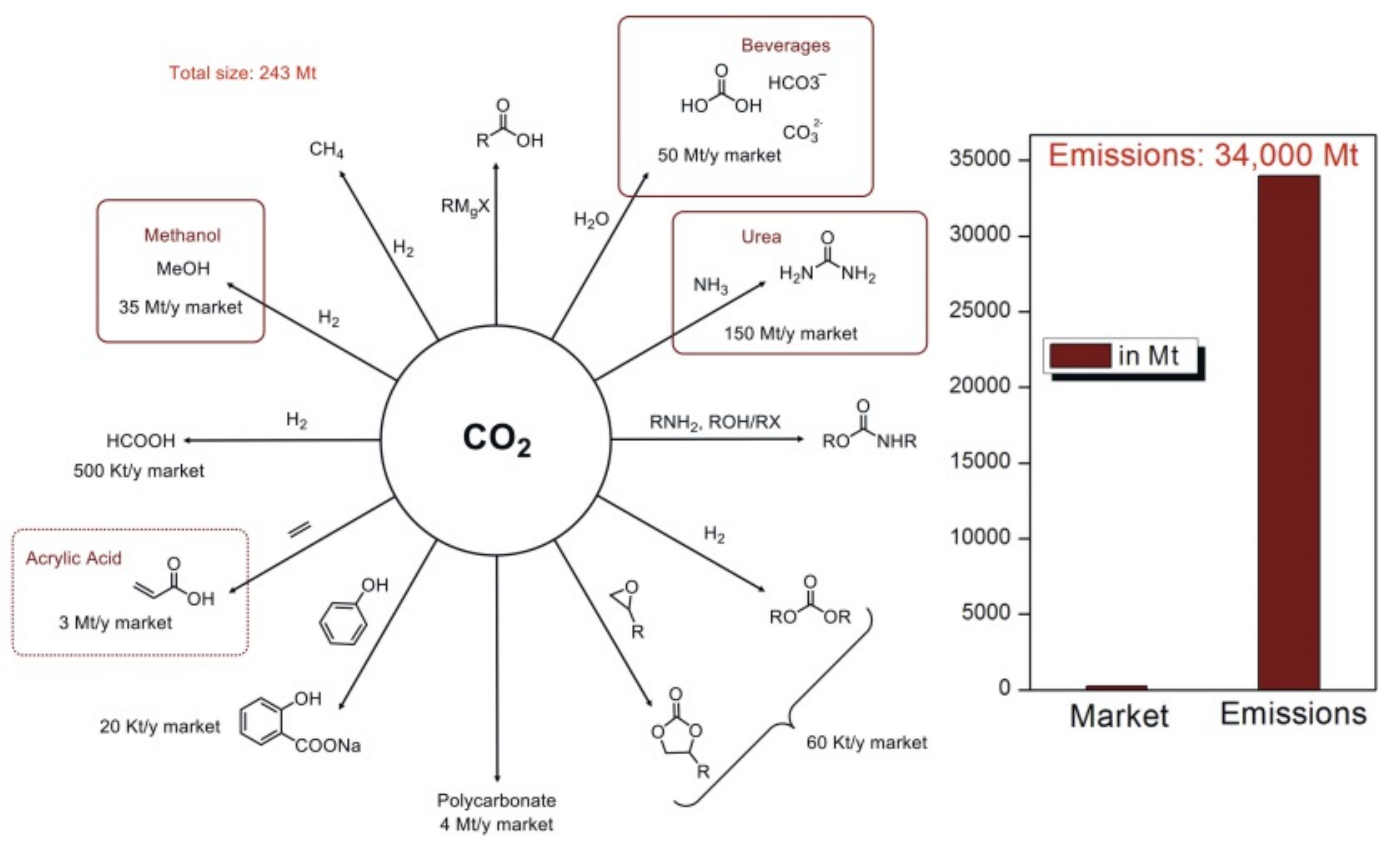


tures, reaching their highest selectivity value of 288 at $50{ }^{\circ} \mathrm{C}$, thus challenging state-of-the-art $\mathrm{CO}_{2}$ separation technologies. ${ }^{[4]}$ We explained this phenomenon by a new concept we have called ' $\mathrm{N}_{2}$-phobicity'.[12] Recently, we have also shown ${ }^{[13]}$ that $\mathrm{CO}_{2} / \mathrm{N}_{2}$ selectivity in these POPs can be further enhanced by incorporating $\mathrm{CO}_{2}$-philic functionalities. In addition, considering the rich chemistry of azo-benzenes, we have synthesized[14] 'chemically similar' azo-polymers using different polymerization routes to clearly identify the origin of $\mathrm{N}_{2}$-phobicity, which was shown to be highly dependent on particle morphology and textural properties. We note that the initial efforts in the area of POPs mostly focused on functional groups effects, thus these findings are rather important to show the impact of textural properties on the gas affinity even for the same chemical functionality.

Recently, we advanced our research efforts in this area by synthesizing (Fig. 3) ultramicroporous POPs incorporating benzothiazole moieties using elemental sulfur (BTAPs) in the absence of any solvent or catalyst. ${ }^{[15]}$ Considering that elemental sulfur is a by-product of natural gas purification, this line of research demonstrates the recycling of $\mathrm{S}_{8}$ into materials for the separation of $\mathrm{CO}_{2}$ from natural and/or landfill gas, thus opening up new directions for the utilization of elemental sulfur, which is a low-value and abundant chemical commodity, for the development of highvalue polymeric materials. BTAPs showed Brunauer-EmmettTeller (BET) surface areas up to $750 \mathrm{~m}^{2} \mathrm{~g}^{-1}$ and were found to be thermally stable up to $550{ }^{\circ} \mathrm{C}$ under $\mathrm{N}_{2}$ and $500{ }^{\circ} \mathrm{C}$ under air conditions. We have observed sulfur loadings up to $24 \mathrm{wt} \%$, which naturally increases $\mathrm{CO}_{2}$ affinity while limiting $\mathrm{CH}_{4}$ diffusion, thus offering a very unique porous material for the purification of $\mathrm{CH}_{4}$ from natural gas and landfill gas streams. We have also developed POPs incorporating molecular cages/macrocycles ${ }^{[16]}$ as monomeric units and showed that their intrinsic properties such as their ability to bind guest molecules in solution can be transferred into the solid-state for the affinity-based separation of complex gas mixtures. For example, the incorporation of triazine-based cages into the POPs allowed us to introduce a so-called 'cage effect' to control the affinity of $\mathrm{CO}_{2}$ towards the sorbent, leading to $15 \mathrm{~kJ}$ $\mathrm{mol}^{-1}$ increase in $\mathrm{CO}_{2}$ binding enthalpy compared to the control polymer without cage units.

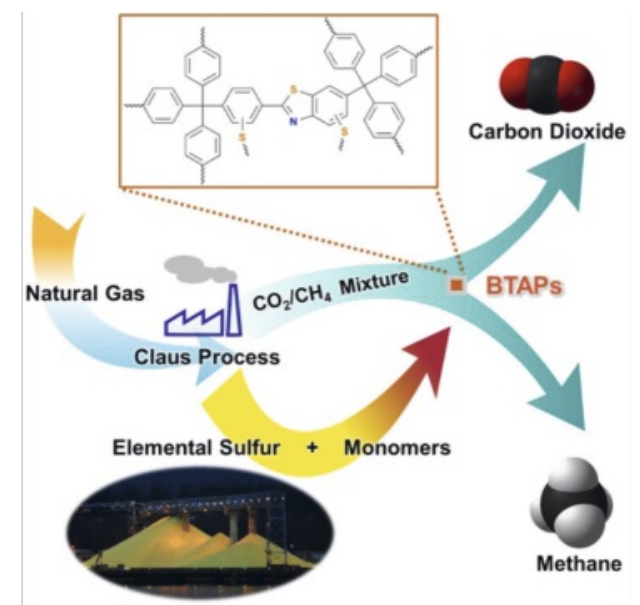

Fig. 3. Elemental sulfur-mediated synthesis of porous organic polymers for $\mathrm{CO}_{2}$ separation from natural gas and flue gas streams. Figure reproduced with permission of the Publisher. ${ }^{[15]}$

\subsection{Porous Organic Polymers for $\mathrm{CO}_{2}$ Capture and Conversion}

Our primary target in this area is to develop porous organocatalysts capable of simultaneously capturing and converting $\mathrm{CO}_{2}$ into value-added products. Accordingly, we have synthesized $^{[17]}$ POPs incorporating $N$-heterocyclic carbenes (Fig. 4).
These polymers exhibited high surface areas and showed very high affinity towards $\mathrm{CO}_{2}$, thus enabling its capture directly from air, thanks to the super basic nature of carbene moieties. We also tested the conversion of $\mathrm{CO}_{2}$ to cyclic carbonates through atom economy reaction under ambient pressure. We have observed exclusive product selectivity towards cyclic carbonates and high conversion yields under these conditions. We have also explored cationic polymers incorporating nucleophilic counteranions for $\mathrm{CO}_{2}$ conversion. ${ }^{[18]}$ In particular, bipyridinium-based POPs with chloride counteranions were shown to be most effective for $\mathrm{CO}_{2}$ conversion.
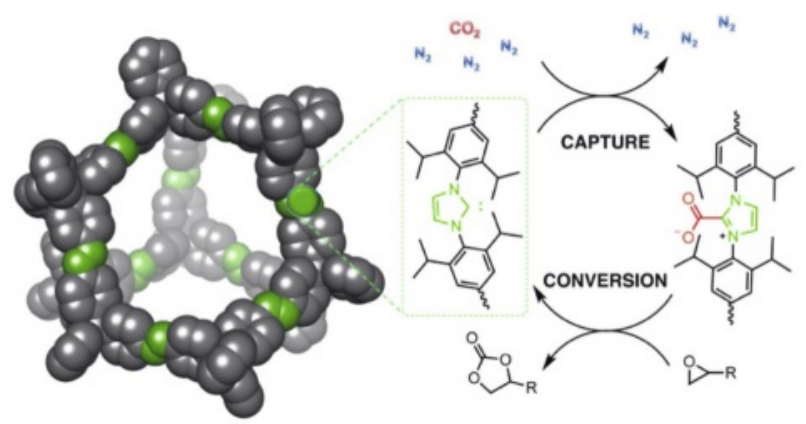

Fig. 4. The chemical structure and proposed capture\&conversion mechanism of porous organic polymer incorporating $N$-heterocyclic carbene moieties. Figure reproduced with permission of the Publisher. ${ }^{[17]}$

More recently, in an effort to improve mass transport kinetics for the catalytic conversion, we have also developed[19] porous cationic polymers with hierarchical porosity - that is the presence of interconnected micro-, meso- and macropores - which showed improved conversion yields for bulky epoxide substrates. Our research efforts in this area are currently focused towards the integration of catalytically active POPs or metal nanoparticles onto two-dimensional membranes to realize simultaneous $\mathrm{CO}_{2}$ separation and conversion. We also explored MOFs with hierarchical porosity by using porous carbon templates ${ }^{[20]}$ or porous metal oxides, ${ }^{[21]}$ in which we controlled surface functionalities to tune the strength of interfacial interactions. We primarily used ZIF- 8 due to its ease of synthesis and catalytic activity for the conversion of $\mathrm{CO}_{2}$ into cyclic carbonates. Importantly, we observed much improved catalytic activity for these composite structures

\subsection{Continuous MOF Membranes for $\mathrm{CO}_{2}$ Separation}

The synthesis of MOF membranes can be achieved by a wide variety of methods including solvent evaporation diffusion, hydro/solvothermal methods, microwave-assisted synthesis and ultrasonic methods. ${ }^{[22]}$ In general, 'in situ growth' or 'seeding and secondary growth' procedures were adopted to fabricate MOF crystals on various substrates. The presence of pinhole defects, grain boundary defects and intercrystalline cracks, however, can significantly reduce the separation performance of MOF membranes. Since the developed methods have a high methodological dependency on the types of substrates, it is hard to control the formation of cracks in large-scale production, which is one of the crucial barriers that postpone the application of MOF membranes in practical applications. In an effort to tackle this problem, we introduced (Fig. 5) the interfacial templation concept, ${ }^{[23]}$ in which graphene oxide was used as a template on the anodic aluminum oxide (AAO) substrate to grow HKUST-1.

The significance of this approach originates from its broad applicability to various substrates. We were able to show the formation of a continuous and crack-free MOF membrane structure, which could also, in principle, address the brittleness of inorganic thin-film membranes based on MOFs. 


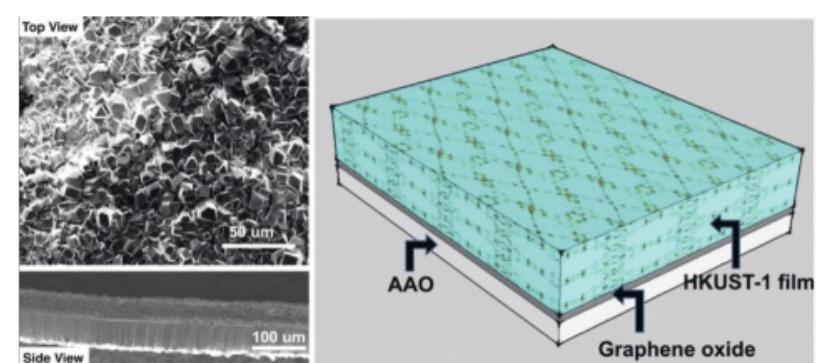

Fig. 5. The graphical representation of continuous MOF films prepared by interfacial templation approach. Figure reproduced with permission of the Publisher. ${ }^{[23]}$

\section{Supramolecular Polymers for High Capacity Electrodes in Li-ion Batteries}

Our research activities in the area of Li-ion batteries focus on the development of high energy density anode and cathode materials. In the area of Li-S batteries, we introduced the concept of elemental sulfur-mediated in situ polymerization to form twodimensional conjugated polymers. This approach allowed us to homogenously distribute high amounts of sulfur (up to $86 \mathrm{wt} \%$ ) within the polymer backbone and led to stable cycling capacity. In addition, we also developed supramolecular polymers in an effort to introduce self-healing effect to the electrodes based on silicon anodes, which in turn, allowed us to achieve exceptional cycling stabilities. Notably, we revealed that it is, in fact, possible to benefit from various supramolecular interactions to alleviate capacity decay pathways for anode materials such as silicon suffering from large volume expansion. In addition, we have recently demonstrated that two-dimensional polymers such as covalent triazine frameworks (CTFs) can be used as artificial SEI layers to stabilize the surface of Li metal anodes.

\subsection{Elemental Sulfur-mediated Polymer Synthesis for Li-S Batteries}

Li-S batteries are considered as next-generation energy storage systems due to their light-weight, low-cost and high gravimetric capacity. However, the major obstacle that still remains (Fig. 6A) is the dissolution of Li-polysulfide intermediates and their shuttling during electrochemical reactions, which leads to a significant capacity decay over cycling. Moreover, the insulating nature of sulfur is also an important challenge. ${ }^{[24]} \mathrm{Li}$-polysulfides can be targeted (Fig. 6B) either through $\mathrm{Li}^{+}$(a hard acid) or sulfur anion (a soft base) by engaging various supramolecular interactions. ${ }^{[25]}$ In this direction, we have demonstrated ${ }^{[26]}$ (Fig. 6C) the synthesis of a CTF with chemically confined sulfur and a high sulfur content of $62 \mathrm{wt} \%$ under catalyst- and solvent-free reaction conditions from 1,4-dicyanobenzene and elemental sulfur. Our synthetic approach introduced a new way of preparing CTFs under environmentally benign conditions by the direct utilization of elemental sulfur. We were able to show that the homogeneous sulfur distribution due to the in situ formation of the polymer network, and chemical sulfur impregnation within the micropores of CTF as well as the presence of hetero atoms effectively suppress the dissolution of polysulfides into the electrolyte. Furthermore, the conjugated nature of the resulting framework facilitates electron and ion transport, which leads to a high-performance lithium-sulfur battery. Notably, when compared to sulfur-doped porous carbon, we observed a significant improvement in the electrochemical performance of the Li-S cell. In order to realise sulfur contents above $80 \mathrm{wt} \%$, we recently demonstrated
A
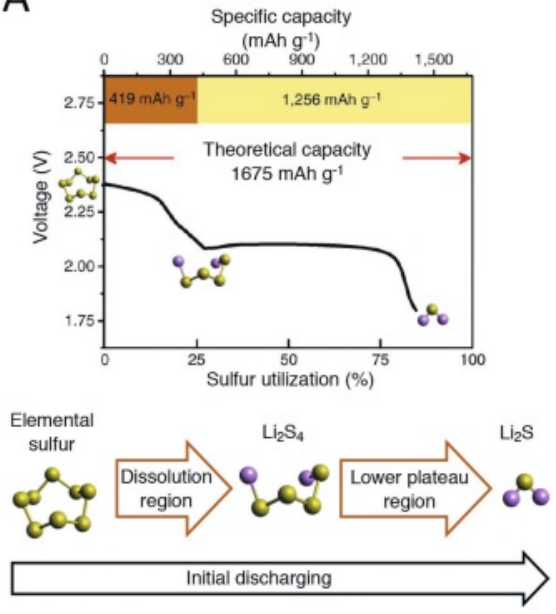

C

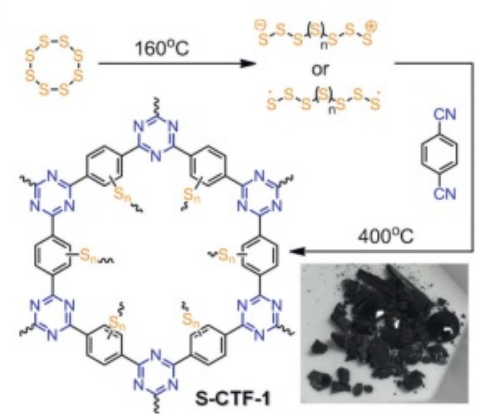

62 wt\% Sulfur
B

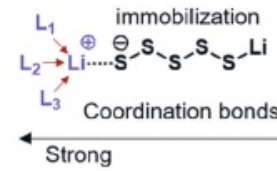

Binding to $\mathrm{Li}^{+}$(Hard Acid)

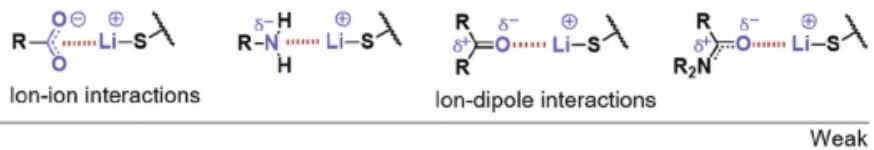

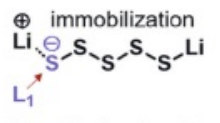
$\mathrm{Ti}_{4} \mathrm{O}_{7}$

Binding to $\mathrm{S}^{-}$(Soft Base)
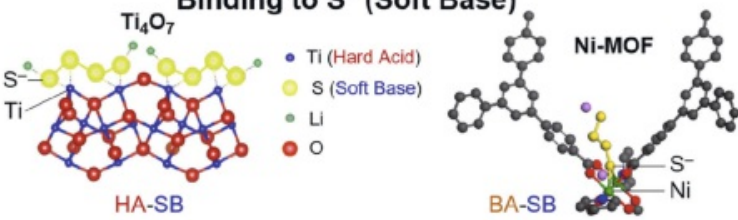

- Ni (Borderline Acid) S (Soft Base) - o - Li - $N$

Coordination bonds

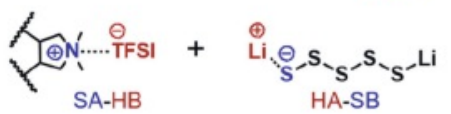
Anion Metathesis

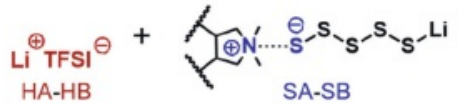
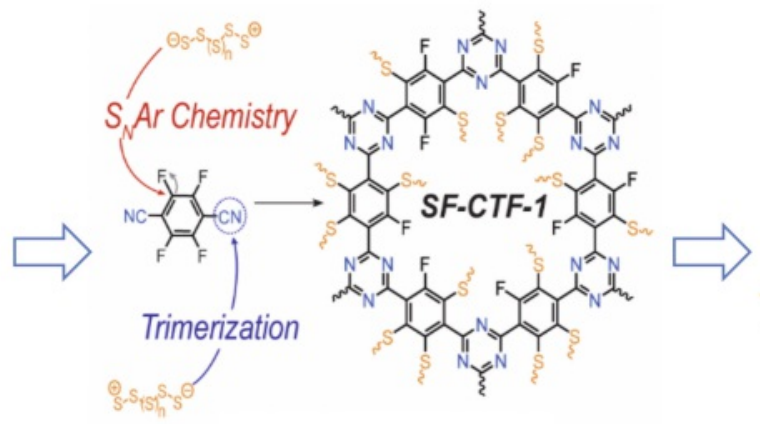

$86 w t \%$ Sulfur

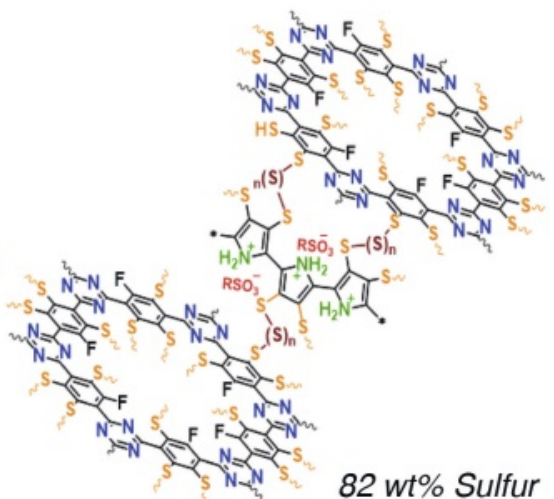

Fig. 6. A) Typical discharge profile of Li-S batteries demonstrating the dissolution region and various redox processes involved during lithiation. B) Various supramolecular approaches to target Li-polysulfides and (C) our synthetic approach for organosulfur polymers with high sulfur contents based on elemental sulfur-mediated synthesis of covalent triazine frameworks. Figure reproduced with permission of the Publisher. ${ }^{[25]}$ 
(Fig. 6C) chemical impregnation of elemental sulfur via $\mathrm{SN}_{\mathrm{Ar}}$ chemistry[27] and C-S insertion. ${ }^{[28]}$ In particular, $\mathrm{SN}_{\mathrm{Ar}}$ chemistry facilitated the dehalogenation and nucleophilic addition reactions of perfluoroaryl units with nucleophilic sulfur chains, achieving a high sulfur content of $86 \mathrm{wt} \%$ in the resulting CTF. The SFCTF, exhibited a specific capacity of $1138.2 \mathrm{mAh} \mathrm{g}^{-1}$ at $0.05 \mathrm{C}$, initial Coulombic efficiency of $93.1 \%$, and capacity retention of $81.6 \%$ after 300 cycles, by utilizing homogeneously distributed sulfur within the micropores and nitrogen atoms of triazine units, thus offering high binding affinity toward lithium polysulfides. Relatively low conductivity of CTF backbone, however, limited both sulfur mass loading and the rate performance of resulting electrodes. To address this challenge, we combined 1D charged polypyrrole with $2 \mathrm{D} \mathrm{CTF}$ in the presence of sulfur to form a $3 \mathrm{D}$ network with high electrical and ionic conductivity as well as suitable charged docking sites on the backbone of polypyrrole for the polysulfide anions. ${ }^{[29]}$ The resulting polymer network enabled stable cycling performance for sulfur mass loadings up to $4.0 \mathrm{mg}_{\text {sulfur }} \mathrm{cm}^{-2}$.

\subsection{Supramolecular Polymeric Binders for Si Anodes in Li-ion Batteries}

We recognized the potential of supramolecular chemistry as a general strategy for solving the capacity-fading problem associated with silicon anodes, an approach which offers extremely high battery capacity compared to conventional graphite-based anodes. Compared to one lithium per six carbon atoms in the fully lithiated state of graphite, silicon can accommodate up to 4.4 $\mathrm{Li}$ atoms, giving rise to a theoretical capacity of $4200 \mathrm{mAh} \mathrm{g} \mathrm{g}^{-1}$. This process, however, leads to a significant volume expansion ( $300 \%)$, causing particle pulverization, morphology change and loss of electrical contact and an unstable solid electrolyte interface (SEI). ${ }^{[11]}$ Our research in this area focuses on the development of supramolecular polymeric binders to alleviate these challenges. We have systematically investigated various noncovalent interactions along with polymer structures - namely, linear, hyperbranched, graft and network polymers and correlated with the electrochemical performance. For example, our investigation ${ }^{[30]}$ of one-dimensional supramolecular polymeric binders incorporating Meldrum's acid revealed that the most critical binder property for silicon anodes in lithium ion batteries is the self-healing effect facilitated by a series of noncovalent interactions such as ion-dipole ${ }^{[31]}$ and host-guest complexation. ${ }^{[32]}$ These investigations suggested the importance of covalent crosslinking while maintaining dynamic nature of supramolecular interactions. Thus, we turned our attention to mechanically interlocked molecules in the form of rotaxanes. In this direction, we demonstrated ${ }^{[33]}$ stable cycling performance for the full cell with an areal capacity of 2.88 $\mathrm{mAh} \mathrm{cm}{ }^{-2}$ for Si microparticle anodes by engaging polyrotaxanebased polymeric binders (Fig. 7).

\section{Working Principle of a Molecular Pulley Binder}
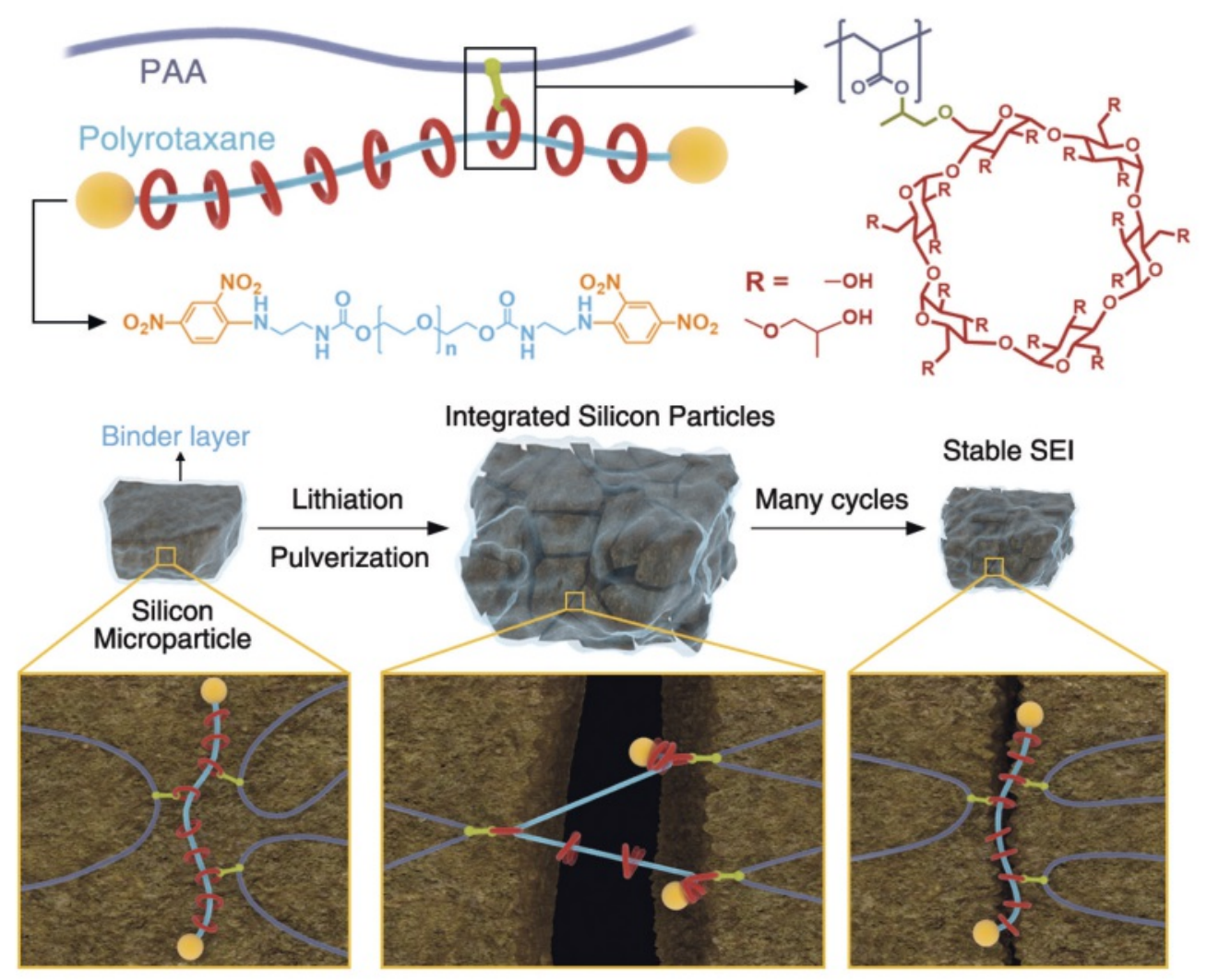

Fig. 7. Chemical structure of a polymeric binder based on 5 wt \% polyrotaxane and PAA. Comparison of the working principle of molecular pulley binder and conventional binders. Figure reproduced with permission of the Publisher. ${ }^{[33]}$

\section{Limitation of Conventional Binders}

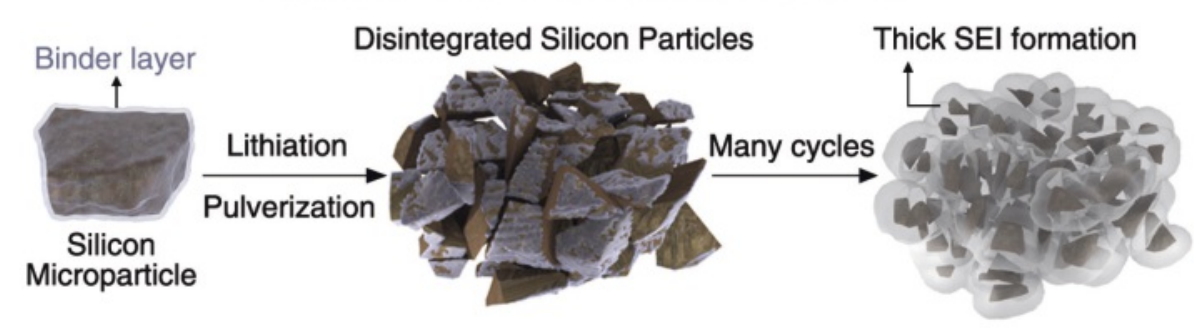


We were able to show that the incorporation of polyrotaxane to conventional polyacrylic acid binder imparts extraordinary elasticity to the polymer network originating from the ring sliding motion of polyrotaxane and this unique binder combination keeps even pulverized silicon particles coalesced without disintegration, enabling stable cycle life for silicon microparticle anodes at commercial-level areal capacities.

\subsection{Supramolecular Polymers as Artificial SEI Layers for Li-metal Anodes}

The Li-metal anode, with the lowest electrochemical potential $(-3.04 \mathrm{~V}$ vs SHE) and the highest theoretical specific capacity $\left(3860 \mathrm{mAh} \mathrm{g}^{-1}\right)$, has aroused discernable attention. ${ }^{[11]}$ However, the uneven growth of Li dendrites during cycling has impeded the practical cell settings, causing an unstable SEI layer, electrolyte consumption, poor CE stability and safety risks by short-circuits. The uncontrolled Li plating and interface stability could be guided and enhanced by a lithiophilic polymer layer. In this direction, our group has introduced (Fig. 8) a 2D polymer (CTF) with rationally designed binding sites for $\mathrm{Li}^{+}$ions to suppress $\mathrm{Li}$-dendrite growth and improve the electrochemical performance. ${ }^{[34]}$

Our strategy for the CTF synthesis involved the cyclotrimerization of 1,4-dicyanobenzene using LiTFSI as a catalyst under ionothermal conditions and the in situ generation and homogeneous distribution of $\mathrm{LiF}$ particles across the framework during synthesis. After loading on the airlaid-paper (AP), as a host to store $\mathrm{Li}$, uniform Li plating was achieved over the entire electrode by the lithiophilicity of CTF originating from the triazine moieties and interface stabilization by LiF. Based on this synergistic effect, the resulting Li-metal anode exhibited ultra-stable $\mathrm{CE}$ at 10 $\mathrm{mA} \mathrm{cm}{ }^{-2}$ for 220 cycles and long lifetime at $5 \mathrm{~mA} \mathrm{~cm}^{-2}$ for 700 cycles, giving a useful design principle for Li-metal stabilization and expanding the application of POPs into this emerging battery technology. Our group is currently further expanding this strategy to various dynamic molecular and supramolecular polymers to effectively stabilize the Li-metal anode surface.

\section{Future Perspectives}

The future of porous organic polymers for $\mathrm{CO}_{2}$ capture and conversion is quite promising. There are, however, still significant challenges present to improve their catalytic performance and cost effectiveness. In this direction, the use of POPs as porous organocatalysts or as supports for single-atom catalysis is rather interesting. Aside from $\mathrm{CO}_{2}$, these systems can also be used as heterogeneous catalysts for various chemical transformations although their textural properties should be carefully tailored to realize hierarchical porosity for improved mass transport kinetics. Catalytic two-dimensional membranes featuring catalytically active POPs, MOFs or metal nanoparticles could enable the realization of simultaneous separation and conversion of $\mathrm{CO}_{2}$. Since the membrane will allow constant removal of product(s) from the reaction mixture, it could, in principle, improve the conversion yields. The development of POPs incorporating organic cages/ macroycles for selective separation of complex mixtures as well as the applications of POPs in the area of LIBs are emerging research topics. In the area of lithium ion batteries, supramolecular chemistry is expected to play a major role in addressing the challenges associated with high energy density electrode materials such as sulfur, Li-metal and silicon. In addition, liquid, polymer and solid electrolytes are also expected to benefit significantly from this approach. Our research efforts to achieve these goals are currently in progress.

\section{Acknowledgements}

I would like to thank all former research group members at Korea Advanced Institute of Science and Technology and current research group members at University of Fribourg for their immense contributions to the development of this research program. These projects has received funding from the National Research Foundation of Korea and Swiss National Science Foundation (SNSF-200021-175947 and 200021-188572)

Received: July 19, 2020

[1] a) G.-R. Walther, E. Post, P. Convey, A. Menzel, C. Parmesan, T. J. C. Beebee, J.-M. Fromentin, O. Hoegh-Guldberg, F. Bairlein, Nature 2002, 416, 389; b) P. Friedlingstein, R. A. Houghton, G. Marland, J. Hackler, T. A. Boden, T. J. Conway, J. G. Canadell, M. R. Raupach, P. Ciais, C. Le Quere, Nature Geosci. 2010, 3, 811.

[2] CSIRO, Australian Government Bureau of Meteorology, http://www.cmar. csiro.au/research/capegrim_graphs.html

[3] A. Goeppert, M. Czaun, R. B. May, G. K. S. Prakash, G. A. Olah, S. R. Narayanan, J. Am. Chem. Soc. 2011, 133, 20164.

[4] D. M. D’Alessandro, B. Smit, J. R. Long, Angew. Chem. Int. Ed. 2010, 49 , 6058.

[5] J. P. Ciferno, T. E. Fout, A. P. Jones, J. T. Murphy, Chem. Eng. Progr. 2009, 33.

[6] N. MacDowell, N. Florin, A. Buchard, J. Hallett, A. Galindo, G. Jackson, C. S. Adjiman, C. K. Williams, N. Shah, P. Fennell, Energ. Environ. Sci. 2010, 3, 1645 .

[7] D. M. D’Alessandro, B. Smit, J. R. Long, Angew. Chem. Int. Ed. 2010, 49, 6058.
Fig. 8. Synthetic scheme for the preparation CTF-LiF, electrode structure and the Li dendrites prevention mechanism along with the electrochemical performance in Li-metal anode. Figure reproduced with permission of the Publisher. ${ }^{[34]}$
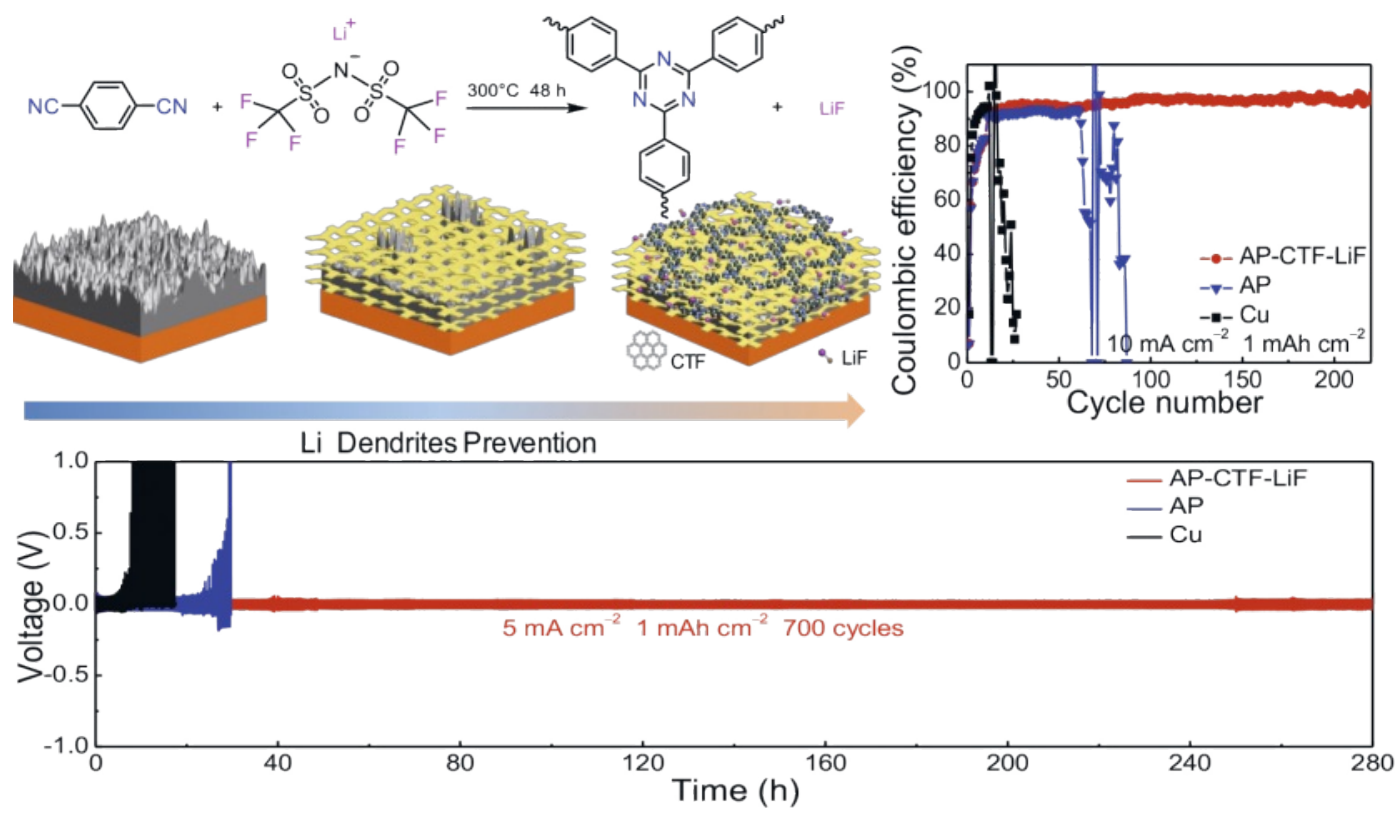
[8] S. Chu, Y. Cui, N. Liu, Nat. Mater. 2017, 16, 16.

[9] https://ccsknowledge.com/pub/Publications/2019Sep_IEA_Putting_CO2_ To_Use.pdf

[10] Q. Liu, L. Wu, R. Jackstell, M. Beller, Nat. Commun. 2015, 6, 5933.

[11] J. W. Choi, D. Aurbach, Nat. Rev. Mater. 2016, 1, 16013.

[12] H. A. Patel, S. Hyun Je, J. Park, D. P. Chen, Y. Jung, C. T. Yavuz, A. Coskun, Nat. Commun. 2013, 4, 1357.

[13] a) J. Byun, S.-H. Je, H. A. Patel, A. Coskun, C. T. Yavuz, J. Mater. Chem. A 2014, 2, 12507; b) H. A. Patel, S. H. Je, J. Park, Y. Jung, A. Coskun, C. T. Yavuz, Chem. Eur. J. 2014, 20, 772.

[14] O. Buyukcakir, S. H. Je, J. Park, H. A. Patel, Y. Jung, C. T. Yavuz, A. Coskun, Chem. Eur. J. 2015, 21, 15320 .

[15] S. H. Je, O. Buyukcakir, D. Kim, A. Coskun, Chem 2016, 1, 482.

[16] a) O. Buyukcakir, Y. Seo, A. Coskun, Chem. Mater. 2015, 27, 4149; b) S. N. Talapaneni, D. Kim, G. Barin, O. Buyukcakir, S. H. Je, A. Coskun, Chem. Mater. 2016, 28, 4460 .

[17] S. N. Talapaneni, O. Buyukcakir, S. H. Je, S. Srinivasan, Y. Seo, K. Polychronopoulou, A. Coskun, Chem. Mater. 2015, 27, 6818.

[18] O. Buyukcakir, S. H. Je, D. S. Choi, S. N. Talapaneni, Y. Seo, Y. Jung, K. Polychronopoulou, A. Coskun, Chem. Commun. 2016, 52, 934.

[19] O. Buyukcakir, S. H. Je, S. N. Talapaneni, D. Kim, A. Coskun, ACS Appl. Mater. Interf. 2017, 9, 7209.

[20] D. Kim, D. W. Kim, W. G. Hong, A. Coskun, J. Mater. Chem. A 2016, 4, 7710 .

[21] M.-K. Kim, D. Kim, J. Y. Seo, O. Buyukcakir, A. Coskun, CrystEngComm 2017, 19, 4147.

[22] S. Qiu, M. Xue, G. Zhu, Chem. Soc. Rev. 2014, 43, 6116

[23] D. Kim, A. Coskun, CrystEngComm 2016, 18, 4013.

[24] Y.-S. Su, Y. Fu, T. Cochell, A. Manthiram, Nat. Commun. 2013, 4, 2985.

[25] T.-w. Kwon, J. W. Choi, A. Coskun, Joule 2019, 3, 662.
[26] S. N. Talapaneni, T. H. Hwang, S. H. Je, O. Buyukcakir, J. W. Choi, A. Coskun, Angew. Chem. Int. Ed. 2016, 55, 3106.

[27] S. H. Je, H. J. Kim, J. Kim, J. W. Choi, A. Coskun, Adv. Func. Mater. 2017 27, 1703947.

[28] S. H. Je, T. H. Hwang, S. N. Talapaneni, O. Buyukcakir, H. J. Kim, J.-S. Yu, S.-G. Woo, M. C. Jang, B. K. Son, A. Coskun, J. W. Choi, ACS Energy Lett. 2016, 1,566 .

[29] J. Kim, A. Elabd, S.-Y. Chung, A. Coskun, J. W. Choi, Chem. Mater. 2020, 32,4185 .

[30] T.-w. Kwon, K. Jeong You, I. Lee, T. S. Kim, W. Choi Jang, A. Coskun, Adv. Mater. 2014, 26, 7979 .

[31] Y. K. Jeong, T.-w. Kwon, I. Lee, T.-S. Kim, A. Coskun, J. W. Choi, Energy Environ Sci. 2015, 8, 1224.

[32] a) Y. K. Jeong, T.-w. Kwon, I. Lee, T.-S. Kim, A. Coskun, J. W. Choi, Nano Letters 2014, 14, 864; b) T.-w. Kwon, Y. K. Jeong, E. Deniz, S. Y AlQaradawi, J. W. Choi, A. Coskun, ACS Nano 2015, 9, 11317.

[33] S. Choi, T.-w. Kwon, A. Coskun, J. W. Choi, Science 2017, 357, 279.

[34] T. Zhou, Y. Zhao, J. W. Choi, A. Coskun, Angew. Chem. Int. Ed. 2019, 58 , 16795.

\section{License and Terms}

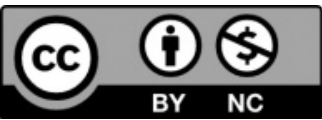

This is an Open Access article under the terms of the Creative Commons Attribution License CC BY_NC 4.0. The material may not be used for commercial purposes.

The license is subject to the CHIMIA terms and conditions: (http:// chimia.ch/component/sppagebuilder/?view=page\&id=12).

The definitive version of this article is the electronic one that can be found at https://doi.org/10.2533/chimia.2020.667 